\title{
Up and downs of a magnetic oblique rotator viewed at high resolution
}

\author{
Y. Nazé ${ }^{1}$, S. A. Zhekov ${ }^{2}$ and A. ud-Doula ${ }^{3}$ \\ ${ }^{1}$ FNRS/Université de Liège, Belgium \\ email: naze@astro.ulg.ac.be \\ ${ }^{2}$ National Astronomical Obsservatory, Bulgaria; ${ }^{3}$ Penn State Worthington Scranton, USA
}

\begin{abstract}
In 2006, the Of?p star HD191612 became the second O-star where a magnetic field was discovered. It provided a benchmark to understand the Of?p phenomenon as a whole. Ten years later, an X-ray monitoring performed at high-resolution reveals the behaviour of the hottest magnetospheric plasma: it is located at $\sim 2 R_{\odot}$, hot but not extreme $(\log (T) \sim 7)$, producing unshifted lines, and displaying a very repetitive variability. A direct comparison with simulations yields an overall good agreement, with only a few further improvements needed.
\end{abstract}

Keywords. X-rays: stars - stars: individual (HD191612) - stars: winds - stars: magnetic fields

The category of Of?p stars gather stars with spectral peculiarities (e.g. narrow emission component in Balmer lines, strong X-ray emission). They are all magnetic and their periodic variations are explained in the context of magnetic oblique rotators (i.e. they are linked to our changing angle-of-view on the magnetically confined winds).

HD191612 (Of?p, $P=537.2 d$ ) was the second magnetic O-star identified. Its X-ray variations occur in phase with $\mathrm{UV} /$ visible changes. To further study them, we gathered Chandra-HETG data at the phases of minimum and maximum fluxes for $196 \mathrm{ks}$ and $142 \mathrm{ks}$, respectively (Nazé et al. 2016). These high-resolution spectra reveal similarities with other magnetic stars $\left(\theta^{1}\right.$ Ori C, HD148937): strong lines from H-like ions, no significant line shift and FWHMs $\sim 400-1200 \mathrm{~km} \mathrm{~s}^{-1}$. The lines appear slightly broader at minimum flux and may be slightly more redshifted at maximum flux but that requires confirmation. The line ratios constrain the temperature to $\log (T) \sim 7$, with a value $25 \%$ smaller at minimum flux. The line ratios also indicate a formation radius $R_{\text {form }}=1.7-2.4 R_{\odot}$, which agree well with the expected Alfven radius. Note that the observed flux modulation (by $\sim 40 \%$ ) shows a great stability over a decade, but cannot be explained by a simple occultation of an axisymmetric equatorial structure at or near this radius, requiring the presence of asymmetries in the confined winds.

A 3D MHD model with the known stellar parameters was computed. It indicates that the wind material should be trapped within 1.6-2.8 $R_{\odot}$, in agreement with observations. The confined winds should however have a temperature $\log (T) \sim 7.5$, larger than observed. Finally, the model predict very narrow line profiles but with broad wings (which are slightly broader at maximum flux). The average DEM from this model was entered into Xspec and directly fitted to the data. A scaling factor of 0.15-0.28 was needed (probably due to a lower $\dot{M}$ ), but an overall good fit was achieved: the broad-band fit is adequate up to $3 \mathrm{keV}$ (but there is too much harder flux, as could be expected from the higher plasma temperature), and the lines are well fitted by both minimum flux and maximum flux profile models - though an even better fit can be achieved with simple Gaussian broadening (i.e. observed lines are slightly broader than predicted).

\section{Reference}

Nazé, Y., ud-Doula, A., \& Zhekov, S. A. 2016, ApJ, 831, 138 